\title{
Application of the Linear Network Model of Finding the Shortest Way of Evacuation of the Population
}

\author{
Vera S. Aslamova \\ Irkutsk State University of Railway Transport \\ Irkutsk, Russia \\ aslamovav@yandex.ru
}

\author{
Elena A. Temnikova \\ Irkutsk State University of Railway Transport \\ Irkutsk, Russia \\ temnikova_ea@bk.ru
}

\begin{abstract}
The report considers an algorithm for solving the problem of advance evacuation of the population, which is formulated as finding the shortest path in a linear network model representing the routes of movement along the existing transport network of roads with a cycle. The starting point is the prefabricated evacuation point, and the final one is the receiving evacuation point, the numbers on the edges are the length of the path between the intermediate points.
\end{abstract}

Keywords-evacuation point, linear network model with a cycle, evacuation of the population, algorithm for finding the shortest path, graph.

\section{THE APPLICATION OF GRAPH THEORY}

At the present stage of development of information technologies, the use of graph theory is wide and diverse:

- in chemistry to describe the structures of chemical elements and the number of theoretically possible isomers of hydrocarbons and other organic compounds [1];

- in computer science and programming (graph flowchart of the program algorithm);

- in communication and transport systems to find the shortest path on the road network [2]. In particular, for routing data on the Internet. Shortest path finding algorithms are used to search for paths between physical objects on Google maps or OpenStreetMap;

- in discrete mathematics [3] for analysis and synthesis of various discrete converters: functional blocks of computers, program complexes, etc ;

- in geoinformation systems when designing structures, power lines, gas pipelines, etc .;

- in logistics [3];

- when replacing equipment and much more.

\section{MATHEMATICAL MODEL OF THE NETWORK MODEL FOR FINDING THE SHORTEST PATH OF EVACUATION OF THE POPULATION}

According to the Resolution of the Government of the Russian Federation of June 22, 2004, No. 303 "On the Procedure for Evacuating the Population, Material and Cultural Values into Safe Areas", depending on the time and date of holding, types of evacuation of the population are singled out:

- pre-emptive (advance), conducted from the forecast zones of emergency situations (ES);
- emergency (urgent - from the zones of action of the damaging factors of the ES) [4]. The routes for evacuating the population, entering the forces and means of eliminating the emergency will be the roads of the existing transport network, the most favorable for traffic. To shorten the time of evacuation, it is necessary to choose a route having a minimum (shortest) extent $[5,6]$.

Consider the task of finding the shortest path of evacuation, which consists in finding the interconnected roads on the transport network, having in aggregate the minimum length from the initial combined evacuation point to the destination (receiving evacuation point). Such a network of roads is represented as a graph with positive weights that correspond to the length of this section, km (Fig. 1).

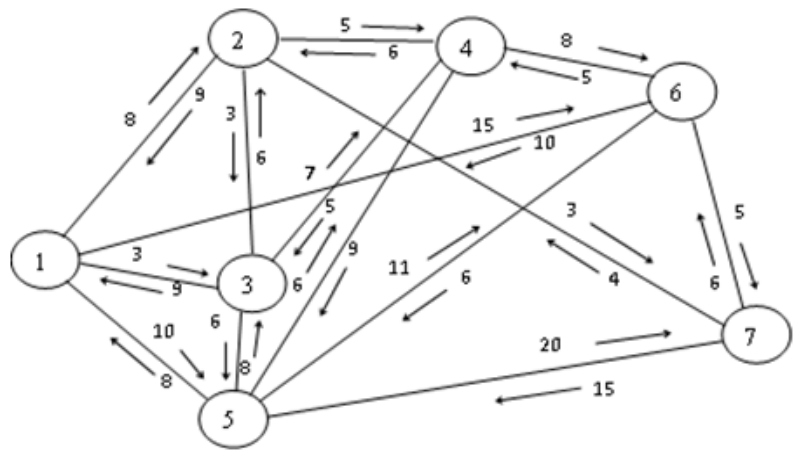

Fig. 1. Linear road network with a cycle: 1 - initial prefabricated evacuation point, 2-6 - intermediate points, 7 - receiving evacuation point. (figure caption)

The model of the shortest path problem is constructed based on the following assumptions:

1. Each variable corresponds to only one arc [7]. [9].

2. Each restriction corresponds to the top of the network

Denote $x_{i j}$ is the value of the flow along the arc between the vertices $(i, j), d_{i j}$ is the arc length. Then the mathematical model of the problem of the shortest path in a network with $n$ nodes is written as [8]:

$$
Z=\sum_{i=1}^{n} \sum_{j=1}^{n} d_{i j} \cdot x_{i j} \rightarrow \min
$$

under constraints 
$\left\{\begin{array}{l}\sum_{j=2}^{n} x_{1 j}=1 \text { (initial prefabricated evacuation point) } \\ \sum_{i=1}^{n} x_{i k}=\sum_{j=1}^{n} x_{k j} \text { (for all } k \neq 1 \text { or } n, \text { which thread came in, this one went out) } \\ \sum_{i=1}^{n-1} x_{i n}=1 \text { (receiving evacuation point) } \\ x_{i j} \geq 0 \text { (for all } i \text { and } j \text { ) }\end{array}\right.$

The constraints of the presented model correspond to the formulation of the transport problem with intermediate points [9]: the flow unit is delivered from node 1 to node $n$. In the example, $n=7$. The first and last constraints establish that the total stream leaving node 1 is 1 , as is the total stream arriving at node $\mathrm{n}$. In any intermediate node, the total input stream is equal to the total output stream [10]. It should be noted that, due to the presence of one-way motion, the quantities $d_{i j}$ and $d_{j i}$ can differ from each other.

\section{AN ALGORITHM FOR FINDING THE SHORTEST PATH OF POPULATION EVACUATION IN A NETWORK WITH A CYCLE}

Let $v_{j}$ be the sum of the lengths of the arcs that form the chain leading from node 1 to node $j, u_{i}$ is the shortest distance from node 1 to node $i$. We set $v_{1}=0$ and $u_{i}=v_{j}$ if $i=j$. Provided that $\mathrm{i}$ and $\mathrm{j}$ are connected by an arc $\left(d_{i j} \neq 0\right)$, the value of $v_{j}$ is determined by the formula

$$
v_{j}=\min \left\{u_{i}+d_{i j}\right\}
$$

If the arc is oriented (that is, the motion is one-sided), the distance in the other direction is assumed to be equal to infinity.

$$
\text { 1. } \quad i=1 \text { and } v_{1}=u_{1}=0 \text {. }
$$

2. In the cycle $j=2$ to $n$, in order to find the value of $v_{j}$, by the formula (1), the node $i$ is located at the minimal distance $\min =d_{i j}$ up to node $j$.

$$
v_{j}=\min ; u_{j_{-}}=v_{j} \text {. }
$$

3. In the cycle with $i=1$ to $n$ for all $j=1$ to $n$, we refine the values of $\mathrm{vj}$ with allowance for two-way traffic. If the values of vj are correctly calculated, then for all values of $i$ and $j$ the inequality

$$
v_{j}-u_{i} \leq d_{i j}
$$

4. If inequality (2) does not hold, then there is a shorter path between nodes $i$ and $j$. Then we recalculate by the formula $v_{j}=u_{i}+d_{i j}$. We replace $u_{j}=v_{j}$. Go to the next value of the cycle parameter.

If condition (2) is not violated in the enclosed cycle, go to step 5, otherwise go to point 3 .

5. The obtained values of $v_{j}$ determine the shortest distance between node 1 and nodes $j=2,3, \ldots, n$. The length of the shortest path from the collection point 1 to the destination is $Z=v_{n}$. The numbers of nodes of the shortest path will be stored in the array $P(m=1 ; P[m]=n)$. We perform the identification of the nodes of the network that form the shortest path, beginning with node $j=n$.
6. We are looking for the node $i$ that precedes the node $j$, for which equality

$$
u_{i}=v_{i}-d_{i j} \text {. Remember } m=m+1 ; P[m]=i ; j=i \text {. }
$$

7. As long as $j \neq 1$, go to point 6 , otherwise - to point 8 .

8 . In the cycle $k$ from $m$ to 1 with step "-1" print the node numbers $P[k]$ of the shortest path.

The algorithm for finding the shortest path on a road network containing loops is based on recursive computations and is discussed in more detail with numerical examples in [11].

\section{ApProbation OF THE ALGORITHM}

The program for the automated calculation of the shortest path of evacuation is implemented in the algorithmic language C\#. In Fig. 2 shows the main form of the program.

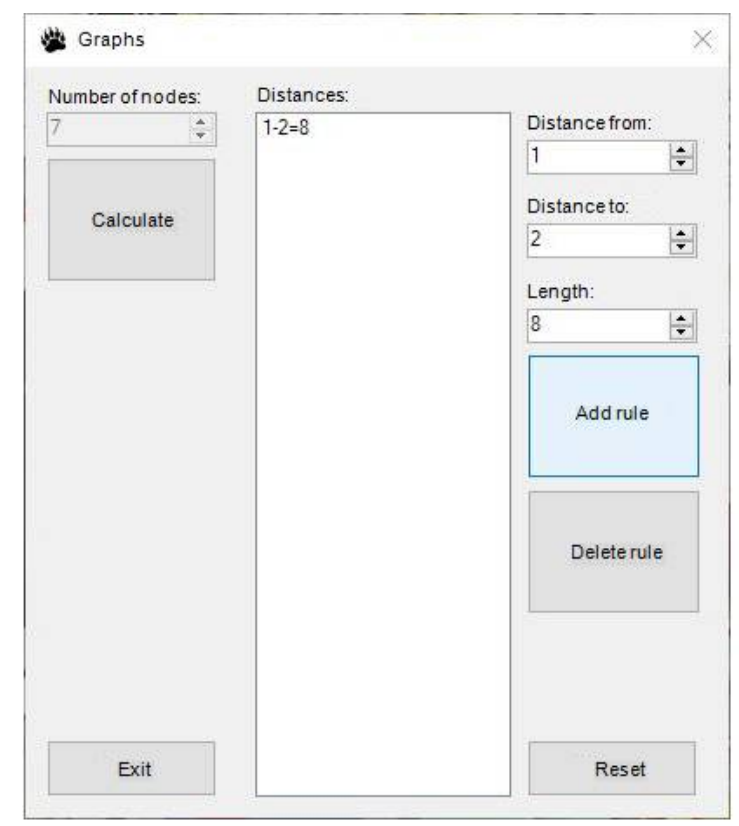

Fig. 2. Dialog box of the program. (figure caption)

To start the program, you need to set the number of network nodes in the upper left corner and enter the distance (arc length) between the selected network node numbers. If the nodes are not connected to each other (there is no arc between nodes), then enter the distance between nodes equal to 0 , and if the arc is oriented (one-sided), then - dial a large number of 32000. After entering the distance, click the "Add rule" button. In case of an error, you can simply specify the same nodes and other distance, the program will find a matching entry and simply replace it, or you can delete the rule completely. After filling the distance matrix, you need to click on the "calculate" button. At the end of the algorithm, the program will give the length of the shortest evacuation path, indicating the route of movement (Fig. 3). 


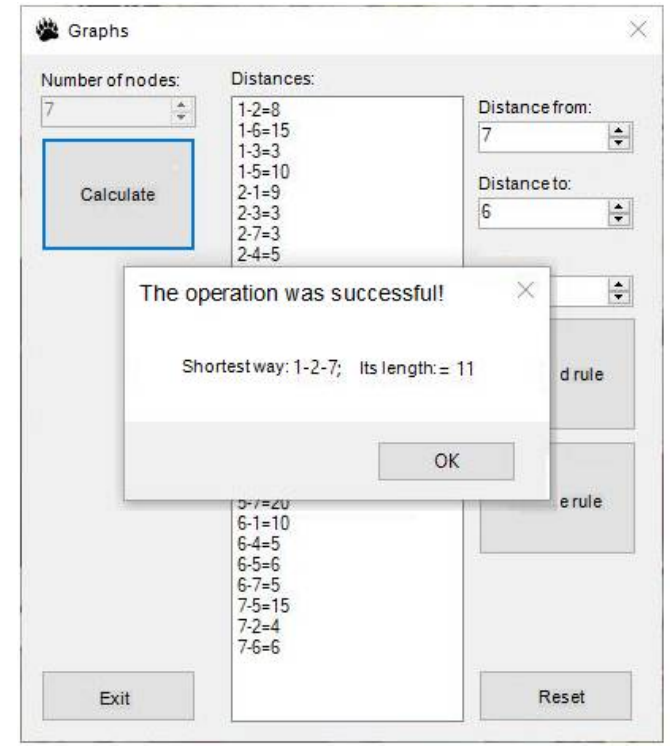

Fig. 3. Results of the program. (figure caption)

The program was tested on the data shown in Fig. 1. Obtained the shortest path, going through the nodes $1 \rightarrow 2 \rightarrow 7$, which has a minimum length of $11 \mathrm{~km}$. The result of the program coincided with the calculation manually.

\section{CONCLUSION}

The developed program will be useful in the compilation of routes of minimum length with the advance evacuation of personnel, the population, material and cultural values from the areas of possible catastrophic flooding, and in case of emergency evacuation in an emergency situation of civil and military time (for example, in case of an accident at a chemically hazardous facility or time of transportation of flammable, explosive and radioactive cargoes). The choice of the shortest evacuation route will save the time of delivery to safe areas, reduce transportation costs and reduce human and material losses.

\section{REFERENCES}

[1] G.S. Yablonsky, V.I .Bykov "Gorban Kinetic models of catalytic reactions". Novosibirsk: Science (Sib. Department), 1983. 255 p.

[2] V.E. Alekseev, V.A. Talanov "Finding the shortest paths in a graph. Counts. Models of calculations. Data structures". Nizhny Novgorod: Nizhny Novgorod state. University, 2005.307 p.

[3] V.I. Pakhomov, G.P. Petrova "Logistics". Moscow: Prospekt, 2006. $232 \mathrm{p}$

[4] Resolution of the Government of the Russian Federation of June 22, 2004 No. 303 "On the procedure for evacuating the population, material and cultural values in safe areas."

[5] Min Manki, Lim Sunho "Segmented arrival graph based evacuation plan assessment algorithm using linear programming". Conference Paper published in Annual IEEE International Systems Conference (SysCon), 2017, pp. 1-8.

[6] Shashi Shekhar, Hui Xiong, Xun Zhou "Dijktra's Shortest Path Algorithm". Encyclopedia of GIS, 2017. 2550 p

[7] Wang Shu-Xi " The Improved Dijkstra's Shortest Path Algorithm and Its Application". Journal Article published in Procedia Engineering, 2012. pp. 1186- 1190 .
[8] Pooja Singal, R.S.Chhillar "Dijkstra Shortest Path Algorithm using Global Position System ". Journal Article published 18 Sep 2014 in International Journal of Computer Application, 2014. pp. 12-18.

[9] Angely Oyola, Dennis G. Romero, Boris X. "Vintimilla A DijkstraBased Algorithm for Selecting the Shortest-Safe Evacuation Routes in Dynamic Environments (SSER)". Lecture Notes in Computer Science, 2017, pp. 131-135.

[10] Kebir IbrahimSeid "AGV Path Planning Using Dijkstra's Routing Algorithm". Journal Article in International Journal of иModern Trends in Engineering \& Research, 2017, pp. 86-93.

[11] V.S. Aslamova, E.A. Temnikova "The theory of making managerial decisions: a textbook". Irkutsk: IrGUPS, 2016. 208 p. 